\section{A Remark on the Stability of Interconnected Nonlinear Systems}

\author{
A. Rapaport and A. Astolfi
}

\begin{abstract}
We use a notion of generalized $L_{2}$-gain for nonlinear systems, where the gain is considered as a function of the state instead of a (global) constant, for characterizing stability properties of interconnected systems. The obtained results generalize the well-known small-gain theorem.
\end{abstract}

Index Terms-Interconnected systems, $L_{2}$-gain, nonlinear systems, small-gain theorem, stability.

\section{INTRODUCTION}

The $L_{2}$-gain analysis of affine nonlinear systems has been widely studied in the literature. For a state-of-the-art, see e.g., [5] and [17] for the smooth case, and [9] for the nondifferentiable one. All these studies deal with the existence of a solution $V$ to a partial differential inequality $\mathcal{H}(V, \gamma) \leq 0$, which reflects the dissipative behavior of the system for a particular supply rate $r(u, y)=\gamma^{2}|u|^{2}-|y|^{2}$, where $u$ and $y$ are respectively the input and output of the system (see [18] for a general framework on dissipativity).

In [10], we have proposed a definition of a generalized $L_{2}$-gain property, where the $L_{2}$-gain constant $\gamma$ is replaced by $\mu=\mu(\cdot)$, a positive function of the state. This new notion of gain stems from the observation that nonlinear systems have nonuniform properties, i.e., the $L_{2}$-gain of a nonlinear system changes with the operating point (this remark has already been made by several authors; see, for instance, [4] and [6]). Our approach is different from the usual ones [18], in the sense that we consider the dissipativity of dynamical systems with respect to supply rates which are functions not only of the norms of the input and the output (as usual) but also of the state of the system: $r(x, u, y)=\mu(x)^{2}|u|^{2}-|y|^{2}$. This allows us to preserve the structure of the Hamiltonian $\mathcal{H}(V, \mu)$ which defines the partial differential inequality, where only $\gamma$ is replaced by $\mu($.$) . Typically, for nonlinear$ systems, $\mu$ cannot be bounded on the whole state space and a finite $L_{2}$-gain cannot be guaranteed in the usual sense (i.e., there does not exist any nonnegative solution to the partial differential inequality with constant $\gamma$ on the whole state space). Nevertheless, one may require that the function $\mu$ is bounded from above by a certain constant $\gamma>0$ when the state of the system evolves outside a neighborhood of the origin (If this neighborhood can be made arbitrarily small, adjusting the function $\mu$, the system is then said to have practical $L_{2}$-gain $\gamma$, see [10]). These concepts are illustrated by several examples in [10], where the practical $L_{2}$ disturbance attenuation problem is also investigated (see also [2] for a complete application).

Other definitions of input-output gains for nonlinear systems have been also proposed in the literature, such as the input-output stability (IOS) [15] (see also integral IOS (iISS) [1], [11], output-to-state stability (OSS) [14] and input-to-state stability with respect to sets [13]), where asymptotic gains are sought as functions of the norm of the input, characterized then by slightly different differential inequalities.

Manuscript received October 19, 2001; revised March 8, 2002, June 12, 2002 , February 7, 2003, and September 22, 2003. Recommended by Associate Editor J. M. A. Scherpen.

A. Rapaport is with the INRA-LASB, Montpellier 34060, France (e-mail: rapaport@ensam.inra.fr).

A. Astolfi is with the Politecnico di Milano, Milan 20133, Italy, and also with Imperial College, London SW7BT, U.K. (e-mail: a.astolfi@imperial.ac.uk).

Digital Object Identifier 10.1109/TAC.2003.821407
In this note, we show that our concept of generalized $L_{2}$-gain provides also some new results concerning the stability of interconnected nonlinear systems, which generalizes in some extent the well known small-gain theorem (see, for instance, [3]).

Several extensions of the small-gain theorem have already been proposed in the literature (see [7] and [16]), where the (constant) gains are replaced by functions of the norm of the input(s). Alternatively, we propose in this note an extension of the small-gain theorem in terms of generalized $L_{2}$ gains.

The note is organized as follows: In Section II, we recall our definition of generalized $L_{2}$-gain property. In Sections III and IV, we present an extension of the small-gain theorem (without input) and then give input-output properties of interconnected systems. Finally, in Section $\mathrm{V}$, we illustrate these new results on an example.

\section{PRACTICAL $L_{2}$-GAin Property}

Consider an input-output dynamical system:

$$
\left\{\begin{array}{l}
\dot{x}=f(x)+k(x) w \\
y=h(x)
\end{array}\right.
$$

in which $x \in \mathbb{R}^{n}, w \in \mathbb{R}^{q}$ and $y \in \mathbb{R}^{s}$ are, respectively, the state, the input, and the output. The functions $f, k$ and $h$ are assumed to be smooth and such that $f(0)=0$ and $h(0)=0$. The notation $x\left(t, x_{0}, w\right)$ stands for the absolutely continuous solution of (1) at time $t>0$ with the initial condition $x(0)=x_{0}$ under the input $w(\cdot)$.

In the following, $\mathcal{O}$ is an open set of $R^{n}, \mu$ a positive function defined on $\mathcal{O}$ and $\gamma$ a positive number.

Definition 1: System (1) has generalized $L_{2}$-gain $\mu$ on $\mathcal{O}$ if there exists a nonnegative storage function $V$, locally bounded on $\mathcal{O}$, such that for any initial condition $x_{0} \in \mathcal{O}$ and input $w \in L_{2}$ we have

$$
\begin{aligned}
\forall T> & 0 \text { s.t. } x\left(t, x_{0}, w\right) \in \mathcal{O} \quad \forall t \in(0, T] \\
V\left(x_{0}\right) \geq & V(x(T)) \\
& -\frac{1}{2} \int_{0}^{T} \mu(x(\tau))^{2}|w(\tau)|^{2}-|y(\tau)|^{2} d \tau .
\end{aligned}
$$

Unlike the linear case, the gain $\mu(\cdot)$ is here sought as a function of the state and not just a constant.The connection with the $L_{2}$-gain in the usual sense is the following.

Definition 2: System (1) has $L_{2}$-gain $\gamma$ on $\mathcal{O}$ if it has generalized $L_{2}$-gain $\mu$ on $\mathcal{O}$ for a certain $\mu$ bounded above by $\gamma$.

Remark 1: The usual $L_{2}$-gain property is exactly recovered with $\mathcal{O}=\mathbb{R}^{n}$

Although the notion of $L_{2}$-gain is defined for nondifferentiable functions [9], only $C^{1}$ conditions shall be considered in the following. For a $C^{1}$ candidate storage function $V$, let $\mathcal{H}(V, \mu)$ be the Hamiltonian function, defined as

$$
\mathcal{H}(V, \mu)(x)=L_{f} V(x)+\frac{\left[L_{k} V(x)\right]^{2}}{2 \mu(x)^{2}}+\frac{|h(x)|^{2}}{2} .
$$

We then recall the key result (see [10]).

Theorem 1: If there exist a $C^{1}$ nonnegative function $V$ and a positive function $\mu$, defined on $\mathcal{O}$, such that

$$
\mathcal{H}(V, \mu)(x) \leq 0 \quad \forall x \in \mathcal{O}
$$

then the system (1) has generalized $L_{2}$-gain $\mu$ on $\mathcal{O}$. 


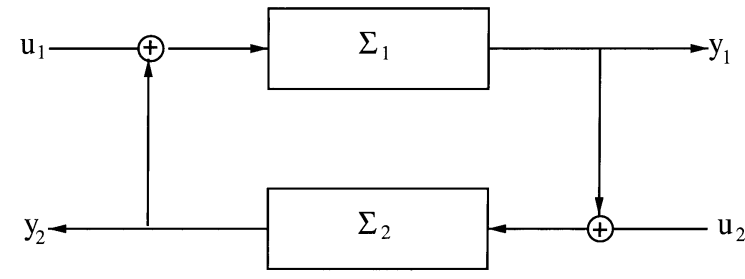

Fig. 1. Interconnection $\Sigma_{1,2}$.

\section{STABILITY OF INTERCONNECTED SYSTEMS}

Our motivation for studying interconnected systems comes from a natural extension of the small gain theorem (see [3]), which claims the stability of the interconnected system when the product of the $L_{2}$-gains of the two subsystems is strictly less than one.

Consider two input-output systems

$$
\Sigma_{i}:\left\{\begin{array}{l}
\dot{x}_{i}=f_{i}\left(x_{i}\right)+k_{i}\left(x_{i}\right) w_{i} \\
y_{i}=h_{i}\left(x_{i}\right)
\end{array} \quad i=1,2\right.
$$

with $\left(x_{1}, w_{1}, y_{1}\right) \in \mathbb{R}^{n_{1}} \times \mathbb{R}^{q} \times \mathbb{R}^{s}$ and $\left(x_{2}, w_{2}, y_{2}\right) \in \mathbb{R}^{n_{2}} \times \mathbb{R}^{s} \times$ $\mathbb{R}^{q}$.

Assume that $\Sigma_{1}$ and $\Sigma_{2}$ have generalized $L_{2}$-gains, respectively $\mu_{1}$ on $\mathcal{O}_{1} \subset \mathbb{R}^{n_{1}}$ and $\mu_{2}$ on $\mathcal{O}_{2} \subset \mathbb{R}^{n_{2}}$, continuous and bounded on their domains, with radially unbounded $C^{1}$ storage functions $V_{1}$ and $V_{2}$, that fulfill the condition (3) of Theorem 1. Denote $\mathcal{H}_{1}$ and $\mathcal{H}_{2}$ their associated Hamiltonian functions, and define the numbers

$$
\gamma_{i}=\sup _{x_{i} \in \mathcal{O}_{i}} \mu_{i}\left(x_{i}\right)<+\infty, \quad i=1,2
$$

Definition 3: Let $\mathcal{S}_{i}$ be a subset of $\mathbb{R}^{n_{i}}$. The system $\Sigma_{i}$ is $\mathcal{S}_{i}$-observable if for any $t \geq 0$, one has the following property:

$$
\begin{aligned}
& \left\{w_{i}(s)=0, y_{i}(s)=0 \quad \forall s \in[0, t]\right\} \\
& \quad \Longrightarrow\left\{x_{i}(s) \in \mathcal{S}_{i} \quad \forall s \in[0, t]\right\} .
\end{aligned}
$$

The system $\Sigma_{i}$ is zero-state observable when $\mathcal{S}_{i}=\{0\}$.

Lemma 2: If $\Sigma_{i}$ is $\left(\mathbb{R}^{n_{i}} \backslash \mathcal{O}_{i}\right)$-observable, then $V_{i}\left(x_{i}\right)>0, \forall x_{i} \in$ $\mathcal{O}_{i}$.

Proof: Take $w_{i}(t)=0, \forall t \geq 0$ and $x_{i, 0} \in \mathcal{O}_{i}$, then

$$
V_{i}\left(x_{i}(t)\right)-V_{i}\left(x_{i, 0}\right) \leq-\frac{1}{2} \int_{0}^{t}\left|y_{i}(\tau)\right|^{2} d \tau
$$

as long as $x_{i}(t) \in \mathcal{O}_{i}$. As $V_{i}\left(x_{i}(t)\right) \geq 0$, we have

$$
V_{i}\left(x_{i, 0}\right) \geq \frac{1}{2} \int_{0}^{t}\left|y_{i}(\tau)\right|^{2} d \tau \text {. }
$$

If $V_{i}\left(x_{i, 0}\right)=0$ then $y(s)=0, \forall s \in[0, t]$ which implies that $x_{i}(s) \in$ $\mathbb{R}^{n_{i}} \backslash \mathcal{O}_{i}, \forall s \in[0, t]$ and contradicts $x_{i, 0} \in \mathcal{O}_{i}$.

The interconnected system $\Sigma_{1,2}$ is the closed-loop system described by the coupling equations (see Fig. 1)

$$
w_{1}=y_{2}+u_{1} \quad w_{2}=y_{1}+u_{2}
$$

with state $x \in \mathbb{R}^{n_{1}+n_{2}}$, input $u \in \mathbb{R}^{s+q}$, and output $y \in \mathbb{R}^{s+q}$

$$
x=\left(\begin{array}{l}
x_{1} \\
x_{2}
\end{array}\right) \quad u=\left(\begin{array}{l}
u_{1} \\
u_{2}
\end{array}\right) \quad y=\left(\begin{array}{l}
y_{1} \\
y_{2}
\end{array}\right) .
$$

Denote $\Gamma=\left\{\left(x_{1}, x_{2}\right) \in \mathcal{O}_{1} \times \mathcal{O}_{2} \mid \mu_{1}\left(x_{1}\right) \mu_{2}\left(x_{2}\right) \geq 1\right\}$.
In this section, we present a result for the stability with no input, in the spirit of the small-gain theorem.

Proposition 3: Assume that $\mathbb{R}^{n} \backslash \mathcal{O}_{i}$ are bounded. Let $u \equiv 0$, then we have the following properties.

1) If $\gamma_{1} \gamma_{2}<1$, the solutions of $\Sigma_{1,2}$ converge toward a bounded limit set. If furthermore $\mathcal{O}_{i}=\mathbb{R}^{n_{i}} \backslash\{0\}$ and $\Sigma_{i}$ are zerostate observable $(i=1,2)$, then $\Sigma_{1,2}$ is globally asymptotically stable.

2) If $\Gamma=\{0\}, \mathcal{O}_{i}=\mathbb{R}^{n_{i}}$ and $\Sigma_{i}$ are zero-state observable ( $i=$ $1,2)$, then $\Sigma_{1,2}$ is globally asymptotically stable.

3) If $\Gamma$ is bounded and if at least one storage function $V_{i}$ is such that

$\gamma_{i}=\max _{x_{i} \in \mathcal{O}_{i}} \mu_{i}$ and $\mathcal{H}_{i}\left(V_{i}, \mu_{i}\right)\left(x_{i}\right) \leq-\left[\mathcal{K}_{i}\left(h_{i}\left(x_{i}\right)\right)\right]^{2} \quad \forall x_{i} \in \mathcal{O}_{i}$

where $\mathcal{K}_{i}$ is a super-linear function (i.e., such that $\left.\lim _{|\xi| \rightarrow \infty}\left|\mathcal{K}_{i}(\xi)\right| /|\xi|=+\infty\right)$, then the solutions of $\Sigma_{1,2}$ converge toward a bounded limit set.

Proof: Consider the candidate Lyapunov function $V\left(x_{1}, x_{2}\right)=$ $V_{1}\left(x_{1}\right)+\lambda V\left(x_{2}\right)$ where $\lambda$ is a positive number (note that $V$ is proper). Then, we have

$$
\begin{aligned}
\dot{V}= & L_{f_{1}} V_{1}\left(x_{1}\right)+L_{k_{1}} V_{1}\left(x_{1}\right) h_{2}\left(x_{2}\right)+\lambda\left(L_{f_{2}} V_{2}\left(x_{2}\right)\right. \\
& \left.+L_{k_{2}} V_{2}\left(x_{2}\right) h_{1}\left(x_{1}\right)\right) \\
\leq & -\frac{\left[L_{k_{1}} V_{1}\left(x_{1}\right)\right]^{2}}{2 \mu_{1}\left(x_{1}\right)^{2}}-\frac{\left|h_{1}\left(x_{1}\right)\right|^{2}}{2}-\lambda \frac{\left[L_{k_{2}} V_{2}\left(x_{2}\right)\right]^{2}}{2 \mu_{2}\left(x_{2}\right)^{2}} \\
& -\lambda \frac{\left|h_{2}\left(x_{2}\right)\right|^{2}}{2}+L_{k_{1}} V_{1}\left(x_{1}\right) h_{2}\left(x_{2}\right) \\
& +\lambda L_{k_{2}} V_{2}\left(x_{2}\right) h_{1}\left(x_{1}\right) \\
= & -\frac{1}{2}\left(\frac{L_{k_{1}} V_{1}\left(x_{1}\right)}{\mu_{1}\left(x_{1}\right)}-\mu_{1}\left(x_{1}\right) h_{2}\left(x_{2}\right)\right)^{2} \\
& -\frac{\lambda}{2}\left(\frac{L_{k_{2}} V_{2}\left(x_{2}\right)}{\mu_{2}\left(x_{2}\right)}-\mu_{2}\left(x_{2}\right) h_{1}\left(x_{1}\right)\right)^{2} \\
& -\frac{\left|h_{1}\left(x_{1}\right)\right|^{2}}{2}\left(1-\lambda \mu_{2}\left(x_{2}\right)^{2}\right) \\
& -\frac{\left|h_{2}\left(x_{2}\right)\right|^{2}}{2}\left(\lambda-\mu_{1}\left(x_{1}\right)^{2}\right) \\
\leq & -\frac{1}{2}\left(1-\lambda \mu_{2}\left(x_{2}\right)^{2}\right)\left|h_{1}\left(x_{1}\right)\right|^{2} \\
& -\frac{1}{2}\left(\lambda-\mu_{1}\left(x_{1}\right)^{2}\right)\left|h_{2}\left(x_{2}\right)\right|^{2}
\end{aligned}
$$

for any $x \in \mathcal{O}_{1} \times \mathcal{O}_{2}$.

1) When $\gamma_{1} \gamma_{2}<1$, we can choose $\lambda$ such that $\lambda>\gamma_{1}^{2}$ and $1 / \lambda>$ $\gamma_{2}^{2}$. From the LaSalle invariance principle, the state $x$ converges toward the smallest $V$-level set that contains $\left(\mathbb{R}^{n_{1}} \backslash \mathcal{O}_{1}\right) \times$ $\left(\mathbb{R}^{n_{2}} \backslash \mathcal{O}_{2}\right)$, which is bounded by hypothesis. When $\mathcal{O}_{i}=$ $\mathbb{R}^{n_{i}} \backslash\{0\}(i=1,2)$, this level set is exactly $V^{-1}(0)$. When the two systems $\Sigma_{i}$ are zero-state observable, then $V^{-1}(0)=\{0\}$ (by Lemma 2) and the global asymptotic stability of $\Sigma_{1,2}$ is then guaranteed.

2) When $\Gamma$ is reduced to $\{0\}$, by continuity of $\mu_{i}$ on $\mathbb{R}^{n_{i}}$, we have

$\left\{\left(x_{1}, x_{2}\right) \in \mathcal{O}_{1} \times \mathcal{O}_{2} \mid \mu_{1}\left(x_{1}\right) \mu_{2}\left(x_{2}\right)=1\right\}=\{(0,0)\}$.

Notice that in this case one has

$$
\gamma_{i}=\max _{x_{i} \in \mathbb{R}^{n_{i}}} \mu_{i}\left(x_{i}\right), \quad i=1,2
$$

and these two maxima are achieved exactly at 0 . Take $\lambda=\gamma_{1}^{2}=$ $\gamma_{2}^{-2}$ and then the right term in the inequality (4) is negative for 
any $x \neq 0$. When the systems $\Sigma_{i}$ are zero-state observable, then by Lemma $2, V^{-1}(0)=\{0\}$, and we conclude the global asymptotic stability of $\Sigma_{1,2}$.

3) Assume that the condition is fulfilled, for instance, for $i=1$. Instead of (4), we have

$$
\begin{aligned}
\dot{V} \leq-\frac{1}{2}\left[\left(1-\lambda \mu_{2}\left(x_{2}\right)^{2}\right)\left|h_{1}\left(x_{1}\right)\right|^{2}+\right. & \left.\mathcal{K}_{1}\left(h_{1}\left(x_{1}\right)\right)^{2}\right] \\
& -\left(\lambda-\mu_{1}\left(x_{1}\right)^{2}\right) \frac{\left|h_{2}\left(x_{2}\right)\right|^{2}}{2}
\end{aligned}
$$

for any $x \in \mathcal{O}_{1} \times \mathcal{O}_{2}$. We then take $\lambda=\gamma_{1}^{2}$ and consider the set

$$
\bar{\Phi}_{1}=\left\{\left(x_{1}, x_{2}\right) \in \mathcal{O}_{1} \times \mathcal{O}_{2} \mid \lambda \mu_{2}\left(x_{2}\right)^{2} \geq 1+\left[\frac{\mathcal{K}_{1}\left(h_{1}\left(x_{1}\right)\right)}{h_{1}\left(x_{1}\right)}\right]^{2}\right\} .
$$

Let us show that $\bar{\Phi}_{1}$ is a bounded set.

The function $\mu_{2}$ being bounded by $\gamma_{2}$, any $x_{1}$ such that $\left(x_{1}, x_{2}\right) \in$ $\bar{\Phi}_{1}$ fulfills $\left[\mathcal{K}_{1}\left(h_{1}\left(x_{1}\right)\right) / h_{1}\left(x_{1}\right)\right]^{2} \leq \gamma_{1}^{2} \gamma_{2}^{2}-1$. The super-linearity of $\mathcal{K}_{1}$ provides then the boundedness of $x_{1}$ on $\bar{\Phi}_{1}$. Take now $\bar{x}_{1} \in \mathcal{O}_{1}$ such that $\mu_{1}\left(\bar{x}_{1}\right)=\gamma_{1}$. Then, any $x_{2}$ such that $\left(x_{1}, x_{2}\right) \in \bar{\Phi}_{1}$ fulfills $\mu_{1}\left(\bar{x}_{1}\right) \mu_{2}\left(x_{2}\right) \geq 1$, i.e., $\left(\bar{x}_{1}, x_{2}\right) \in \Gamma$. The boundedness of $\Gamma$ implies then that $x_{2}$ is bounded on $\bar{\Phi}_{1}$.

Finally, by LaSalle invariance principle, $x(t)$ converges toward the smallest $V$-level set that contains $\bar{\Phi}_{1} \cup\left(\mathbb{R}^{n_{1}} \backslash \mathcal{O}_{1}\right) \times\left(\mathbb{R}^{n_{2}} \backslash \mathcal{O}_{2}\right)$

\section{Remarks 2 .}

i) When systems $\Sigma_{1}, \Sigma_{2}$ have $L_{2}$-gains $\gamma_{1}, \gamma_{2}$ on $\mathbb{R}^{n_{1}}, \mathbb{R}^{n_{2}}$, point 1 is the natural extension of the well known small-gain theorem, that claims the internal stability of interconnected systems with no input when $\gamma_{1} \gamma_{2}<1$.

ii) Points 2) and 3) show clearly the advantage of considering generalized $L_{2}$-gains as we can guarantee the global asymptotic stability even when $\gamma_{1} \gamma_{2}=1$ (see the example in Section V).

\section{INPUT-OUTPUT PROPERTY OF INTERCONNECTED SYSTEMS}

We study now the $L_{2}$ bounded input-bounded output property of the interconnected system $\Sigma_{1,2}$.

Proposition 4: When the input $u$ belongs to $L_{2}$, then the output $y$ has the following properties.

1) If $\gamma_{1} \gamma_{2}<1$, then $y$ belongs to $L_{2}([0, T])$ for any $T>0$ such that $x(t)$ stays in $\mathcal{O}_{1} \times \mathcal{O}_{2}$, for $t \leq T$.

2) If $\Gamma=\{0\}$, then $y$ belongs to $L_{2}([0, T])$ for any $T>0$ such that $x(t)$ stays in $\mathcal{O}_{1} \times \mathcal{O}_{2} \backslash\{0\}$, for $t \leq T$.

3) If $\Gamma$ is bounded and if at least one storage function $V_{i}$ is such that

$\gamma_{i}=\max _{x_{i} \in \mathcal{O}_{i}} \mu_{i}$ and $\mathcal{H}_{i}\left(V_{i}, \mu_{i}\right)\left(x_{i}\right) \leq-\left[\mathcal{K}_{i}\left(h_{i}\left(x_{i}\right)\right)\right]^{2} \quad \forall x_{i} \in \mathcal{O}_{i}$

where $\mathcal{K}_{i}$ is a super-linear function, then the output $y$ belongs in $L_{2}([0, T])$ as long as the state evolves in $\mathcal{O}_{1} \times \mathcal{O}_{2} \backslash \bar{\Phi}_{i}$, where

$$
\begin{aligned}
\bar{\Phi}_{i}=\left\{\left(x_{1}, x_{2}\right) \in \mathcal{O}_{1} \times \mathcal{O}_{2} \mid \gamma_{i}^{2} \mu_{j}\left(x_{j}\right)^{2}\right. & \\
& \left.\geq 1+\left[\frac{\mathcal{K}_{i}\left(h_{i}\left(x_{i}\right)\right)}{h_{i}\left(x_{i}\right)}\right]^{2}\right\} \quad(j \neq i)
\end{aligned}
$$

is a compact set.
Proof: Consider the function $V(x)=V_{1}\left(x_{1}\right)+\lambda V_{2}\left(x_{2}\right)$ where $\lambda$ is a positive number. By hypothesis, $V$ is proper. From the property (2) fulfilled by $V_{1}$ and $V_{2}$, we have

$$
\begin{aligned}
V(x(T))-V(x(0)) \leq & \frac{1}{2} \int_{0}^{T} \mu_{1}\left(x_{1}(\tau)\right)^{2}\left|y_{2}(\tau)+u_{1}(\tau)\right|^{2} \\
& -\left|y_{1}(\tau)\right|^{2}+\lambda\left[\mu_{2}\left(x_{2}(\tau)\right)^{2} \mid y_{1}(\tau)\right. \\
& \left.+\left.u_{2}(\tau)\right|^{2}-\left|y_{2}(\tau)\right|^{2}\right] d \tau \\
\leq & \frac{1}{2} \int_{0}^{T}\left|y_{1}(\tau)\right|^{2}\left[\lambda \mu_{2}\left(x_{2}(\tau)\right)^{2}-1\right] \\
& +2\left|y_{1}(\tau)\right| \lambda \mu_{2}\left(x_{2}(\tau)\right)^{2}\left|u_{2}(\tau)\right| \\
& +\left|y_{2}(\tau)\right|^{2}\left[\mu_{1}\left(x_{1}(\tau)\right)^{2}-\lambda\right] \\
& +2\left|y_{2}(\tau)\right| \mu_{1}\left(x_{1}(\tau)\right)^{2}\left|u_{1}(\tau)\right| \\
& +\mu_{1}\left(x_{1}(\tau)\right)^{2}\left|u_{1}(\tau)\right|^{2} \\
& +\lambda \mu_{2}\left(x_{2}(\tau)\right)^{2}\left|u_{2}(\tau)\right|^{2} d \tau
\end{aligned}
$$

for $T$ such that $x(t) \in \mathcal{O}_{1} \times \mathcal{O}_{2}, \forall t \leq T$. $V$ being nonnegative, we obtain from the previous inequality

$$
\begin{aligned}
& \frac{1}{2} \int_{0}^{T}\left|y_{1}(\tau)\right|^{2}\left[1-\lambda \mu_{2}\left(x_{2}(\tau)\right)^{2}\right] \\
& +\left|y_{2}(\tau)\right|^{2}\left[\lambda-\mu_{1}\left(x_{1}(\tau)\right)^{2}\right] d \tau \\
& \quad-\int_{0}^{T}\left|y_{1}(\tau)\right| \lambda \mu_{2}\left(x_{2}(\tau)\right)^{2}\left|u_{2}(\tau)\right| \\
& +\left|y_{2}(\tau)\right| \mu_{1}\left(x_{1}(\tau)\right)^{2}\left|u_{1}(\tau)\right| d \tau \\
& \quad \leq V(x(0))+\frac{1}{2} \int_{0}^{T} \mu_{1}\left(x_{1}(\tau)\right)^{2}\left|u_{1}(\tau)\right|^{2} \\
& \quad+\lambda \mu_{2}\left(x_{2}(\tau)\right)^{2}\left|u_{2}(\tau)\right|^{2} d \tau .
\end{aligned}
$$

Finally, applying the Cauchy-Schwartz inequality on the second integral of the left term of the previous inequality, we obtain

$$
\begin{aligned}
& \frac{1}{2} \int_{0}^{T}\left|y_{1}(\tau)\right|^{2}\left[1-\lambda \mu_{2}\left(x_{2}(\tau)\right)^{2}\right]+\left|y_{2}(\tau)\right|^{2} \\
& \times\left[\lambda-\mu_{1}\left(x_{1}(\tau)\right)^{2}\right] d \tau \\
& -\lambda\left\|u_{2}\right\|_{[0, T]} \int_{0}^{T}\left|y_{1}(\tau)\right| \mu_{2}\left(x_{2}(\tau)\right)^{2} d \tau \\
& -\left\|u_{1}\right\|_{[0, T]} \int_{0}^{T}\left|y_{2}(\tau)\right| \mu_{1}\left(x_{1}(\tau)\right)^{2} d \tau \\
& \leq V(x(0))+\frac{1}{2} \int_{0}^{T} \mu_{1}\left(x_{1}(\tau)\right)^{2}\left|u_{1}(\tau)\right|^{2} \\
& \quad+\lambda \mu_{2}\left(x_{2}(\tau)\right)^{2}\left|u_{2}(\tau)\right|^{2} d \tau .
\end{aligned}
$$

1) If $\gamma_{1} \gamma_{2}<1$, we choose $\lambda>0$ such that $\lambda-\gamma_{1}^{2} \geq \varepsilon_{1}>$ 0 and $1-\lambda \gamma_{2}^{2} \geq \varepsilon_{2}>0$. Then, the inequality (5) provides

$$
\begin{aligned}
& \frac{\varepsilon_{2}}{2}\left\|y_{1}\right\|_{[0, T]}^{2}-\left\|y_{1}\right\|_{[0, T]}\left\|u_{2}\right\|_{[0, T]}+\frac{\varepsilon_{1}}{2}\left\|y_{2}\right\|_{[0, T]}^{2} \\
& \quad-\lambda\left\|y_{2}\right\|_{[0, T]}\left\|u_{1}\right\|_{[0, T]} \leq V(x(0))+\frac{\lambda}{2}\left\|u_{1}\right\|_{[0, T]}^{2}+\frac{1}{2}\left\|u_{2}\right\|_{[0, T]}^{2}
\end{aligned}
$$

and we conclude that $\|y\|_{[0, T]}$ is bounded as long as $x(t) \in$ $\mathcal{O}_{1} \times \mathcal{O}_{2}, \forall t \leq T$.

2) When $\Gamma=\{0\}$, the maxima of the functions $\mu_{i}$ are reached exactly at 0 , and $\gamma_{1} \gamma_{2}$ is necessarily equal to one. We then take 
$\lambda=\gamma_{1}^{2}=\gamma_{2}^{-2}$. By continuity of the functions $\mu_{i}$ at 0 , we have: $\forall \eta>0, \exists \varepsilon_{1}>0, \exists \varepsilon_{2}>0$ such that

$$
\lambda-\mu_{1}\left(x_{1}\right)^{2} \geq \varepsilon_{1}>0 \text { and } 1-\lambda \mu_{2}\left(x_{2}\right)^{2} \geq \varepsilon_{2}>0
$$

for any $\left(x_{1}, x_{2}\right) \in \mathcal{O}_{1} \times \mathcal{O}_{2} \backslash B(0, \eta)$, and we obtain, as before, that $\|y\|_{[0, T]}$ is bounded as long as $x(t) \in \mathcal{O}_{1} \times \mathcal{O}_{2}$ and $x(t) \notin$ $B(0, \eta)$ for any $t \leq T$. This property is fulfilled for any arbitrary small $\eta>0$.

3) Assume that the condition is fulfilled, for instance, for $i=1$. Then, instead of (5), one can write

$$
\begin{aligned}
& \frac{1}{2} \int_{0}^{T}\left|y_{1}(\tau)\right|^{2}\left[1-\lambda \mu_{2}\left(x_{2}(\tau)\right)^{2}\right] \\
& +\mathcal{K}_{1}\left(y_{1}(\tau)\right)^{2} d \tau-\lambda \gamma_{2}^{2}\left\|y_{1}\right\|_{[0, T]}\left\|u_{2}\right\|_{[0, T]} \\
& +\frac{\lambda-\gamma_{1}^{2}}{2}\left\|y_{2}\right\|_{[0, T]}^{2}-\gamma_{1}^{2}\left\|y_{2}\right\|_{[0, T]}\left\|u_{1}\right\|_{[0, T]} \\
& \quad \leq V(x(0))+\frac{\gamma_{1}^{2}}{2}\left\|u_{1}\right\|_{[0, T]}^{2}+\frac{\lambda \gamma_{2}^{2}}{2}\left\|u_{2}\right\|_{[0, T]}^{2}
\end{aligned}
$$

as long as $x(t) \in \mathcal{O}_{1} \times \mathcal{O}_{2}, \forall t \leq T$. Take $\lambda=\gamma_{1}^{2}+\varepsilon$ with $\varepsilon>0$, and consider the set

$$
\begin{aligned}
\Phi_{1}^{\varepsilon}=\left\{\left(x_{1}, x_{2}\right) \in \mathcal{O}_{1} \times \mathcal{O}_{2} \mid 1-\left(\gamma_{1}^{2}+\varepsilon\right)\right. & \mu_{2}\left(x_{2}\right)^{2} \\
& \left.+\left[\frac{\mathcal{K}_{1}\left(h_{1}\left(x_{1}\right)\right)}{h_{1}\left(x_{1}\right)}\right]^{2}<\varepsilon\right\} .
\end{aligned}
$$

Then, from (6), we conclude, as before, that $\|y\|_{[0, T]}$ stays bounded, as long as $x(t) \in \mathcal{O}_{1} \times \mathcal{O}_{2}$ and $x(t) \notin \Phi_{1}^{\varepsilon}, \forall t \leq T$. This property is fulfilled for any arbitrary small number $\varepsilon$. Finally, $\mu_{2}$ being bounded, we have $\bigcap_{\varepsilon>0} \Phi_{1}^{\varepsilon}=\bar{\Phi}_{1}$, which is is bounded, by compactness of $\Gamma$ and super-linearity of $\mathcal{K}_{1}$.

\section{ILLUSTRATIVE EXAMPLE}

Consider the following interconnected system:

$\Sigma_{1}:\left\{\begin{array}{l}\dot{x}_{1}=-x_{1}-x_{1}^{3}+\alpha_{1} y_{2} \\ y_{1}=x_{1}\end{array}\right.$

$$
\Sigma_{2}:\left\{\begin{array}{l}
\dot{x}_{2}=-x_{2}-x_{2}^{3}+\alpha_{2} y_{1} \\
y_{2}=x_{2}
\end{array}\right.
$$

$\Sigma_{1}$ and $\Sigma_{2}$ have generalized $L_{2}$-gain on $\mathbb{R}$

$$
\mu_{i}\left(x_{i}\right)=\frac{\left|\alpha_{i}\right|}{\sqrt{1+x_{i}^{2}}}, \quad i=1,2
$$

with storage functions $V_{i}\left(x_{i}\right)=x_{i}^{2} / 2(i=1,2)$ as

$$
\mathcal{H}\left(V_{i}, \mu_{i}\right)\left(x_{i}\right)=-\frac{x_{i}^{4}}{2}, \quad i=1,2
$$

thus $\mathcal{K}_{i}\left(y_{i}\right)=\left(y_{i}^{2} / \sqrt{2}\right)$. Furthermore, one has $\left|\alpha_{i}\right|=$ $\max _{x_{i} \in \mathbb{R}} \mu_{i}\left(x_{i}\right)=\mu_{i}(0)$. Then, the set

$$
\Gamma=\left\{\left(x_{1}, x_{2}\right) \in \mathbb{R}^{2} \mid\left(1+x_{1}^{2}\right)\left(1+x_{2}^{2}\right) \leq \alpha_{1}^{2} \alpha_{2}^{2}\right\}
$$

is bounded, whatever are $\alpha_{1}, \alpha_{2}$ and we conclude that $\Sigma_{1,2}$ is stable for zero input.

1) If $\left|\alpha_{1} \alpha_{2}\right|<1$, by the usual small gain theorem, $\Sigma_{1,2}$ is globally asymptotically stable.

2) If $\left|\alpha_{1} \alpha_{2}\right|=1, \Sigma_{1,2}$ is also globally asymptotically stable (although the usual small gain theorem is not conclusive).
3) If $\left|\alpha_{1} \alpha_{2}\right|>1$, we have

$$
\begin{aligned}
& \bar{\Phi}_{1}=\left\{\left(x_{1}, x_{2}\right) \in \mathbb{R}^{2} \mid\left(1+\frac{1}{2} x_{1}^{2}\right)\left(1+x_{2}^{2}\right) \leq \alpha_{1}^{2} \alpha_{2}^{2}\right\} \\
& \bar{\Phi}_{2}=\left\{\left(x_{1}, x_{2}\right) \in \mathbb{R}^{2} \mid\left(1+x_{1}^{2}\right)\left(1+\frac{1}{2} x_{2}^{2}\right) \leq \alpha_{1}^{2} \alpha_{2}^{2}\right\}
\end{aligned}
$$

and we conclude that the state converges toward a bounded set $S$, which is the intersection of two level sets, which are the smallest $V_{1}+\alpha_{1}^{2} V_{2}$ (respectively, $\alpha_{2}^{2} V_{1}+V_{2}$ ) level set containing $\bar{\Phi}_{1}$ (respectively, $\bar{\Phi}_{2}$ ).

When $\left(x_{1}, x_{2}\right) \in \bar{\Phi}_{1}$, we have

$$
\begin{aligned}
& x_{1}^{2} \leq 2\left(\alpha_{1}^{2} \alpha_{2}^{2}-1\right)-x_{2}^{2}\left(2+x_{1}^{2}\right) \quad \text { and } \\
& x_{2}^{2} \leq\left(\alpha_{1}^{2} \alpha_{2}^{2}-1\right)-\frac{1}{2} x_{1}^{2}\left(1+x_{2}^{2}\right) .
\end{aligned}
$$

Thus

$$
\begin{aligned}
V_{1}\left(x_{1}\right)+\alpha_{1}^{2} V_{2}\left(x_{2}\right) \leq \min & {\left[\left(\alpha_{1}^{2} \alpha_{2}^{2}-1\right)\right.} \\
& -\frac{1}{2} x_{2}^{2}\left(2-\alpha_{1}^{2}+x_{1}^{2}\right) \\
& \frac{1}{2} \alpha_{1}^{2}\left(\alpha_{1}^{2} \alpha_{2}^{2}-1\right) \\
& \left.-\frac{1}{4} x_{1}^{2}\left(\alpha_{1}^{2}-2+\alpha_{1}^{2} x_{2}^{2}\right)\right]
\end{aligned}
$$

which is equivalent to

$$
\frac{1}{2} x_{1}^{2}+\frac{1}{2} \alpha_{1}^{2} x_{2}^{2} \leq \max \left(1, \frac{1}{2} \alpha_{1}^{2}\right)\left(\alpha_{1}^{2} \alpha_{2}^{2}-1\right) .
$$

In a similar way, when $\left(x_{1}, x_{2}\right) \in \bar{\Phi}_{2}$, we have

$$
\frac{1}{2} \alpha_{2}^{2} x_{1}^{2}+\frac{1}{2} x_{2}^{2} \leq \max \left(1, \frac{1}{2} \alpha_{2}^{2}\right)\left(\alpha_{1}^{2} \alpha_{2}^{2}-1\right) .
$$

So, the set $S$ has the following representation

$$
S=\left\{\begin{array}{l|l}
\left(x_{1}, x_{2}\right) & \begin{array}{l}
x_{1}^{2}+\alpha_{1}^{2} x_{2}^{2} \leq \max \left(\alpha_{1}^{2}, 2\right)\left(\alpha_{1}^{2} \alpha_{2}^{2}-1\right) \\
\alpha_{2}^{2} x_{1}^{2}+x_{2}^{2} \leq \max \left(\alpha_{2}^{2}, 2\right)\left(\alpha_{1}^{2} \alpha_{2}^{2}-1\right)
\end{array}
\end{array}\right\} .
$$

Simulations has been run for $\alpha_{1}=2$ and $\alpha_{2}=3$. The interconnected system has then two equilibriums which belong to the set $S$, as illustrated on Fig. 2.

Remarks: The systems $\Sigma_{1}, \Sigma_{2}$ in (7) are also ISS; see [12]

$$
\begin{aligned}
\nabla V_{i}\left(x_{i}\right)\left(-x_{i}-x_{i}^{3}+\alpha_{i} u_{i}\right) & \leq-x_{i}^{4} \quad \text { when } \\
\left|x_{i}\right| & \geq\left|\alpha_{i}\right|\left(\left|u_{i}\right|\right), \quad i=1,2 .
\end{aligned}
$$

So, $V_{1}, V_{2}$ are ISS-Lyapunov functions with ISS-gains $\chi_{1}, \chi_{2}$, as functions of the norm of the inputs $u_{1}=y_{2}$ and $u_{2}=y_{1}$

$$
\chi_{i}\left(\left|u_{i}\right|\right)=\left|\alpha_{i}\right|\left|u_{i}\right|, \quad i=1,2 .
$$

Then, according to [7], the global asymptotic stability of the interconnected system is guaranteed when $\chi_{1} \circ \chi_{2}(r)<r, \forall r>0$, i.e., when $\left|\alpha_{1} \alpha_{2}\right|<1$, which does not bring in this example more information than the usual small-gain theorem. On the contrary, the proposed generalization of the small gain theorem allows not only to conclude global asymptotic stability for $\left|\alpha_{1} \alpha_{2}\right|=1$, but also global boundedness for $\left|\alpha_{1} \alpha_{2}\right|>1$. 


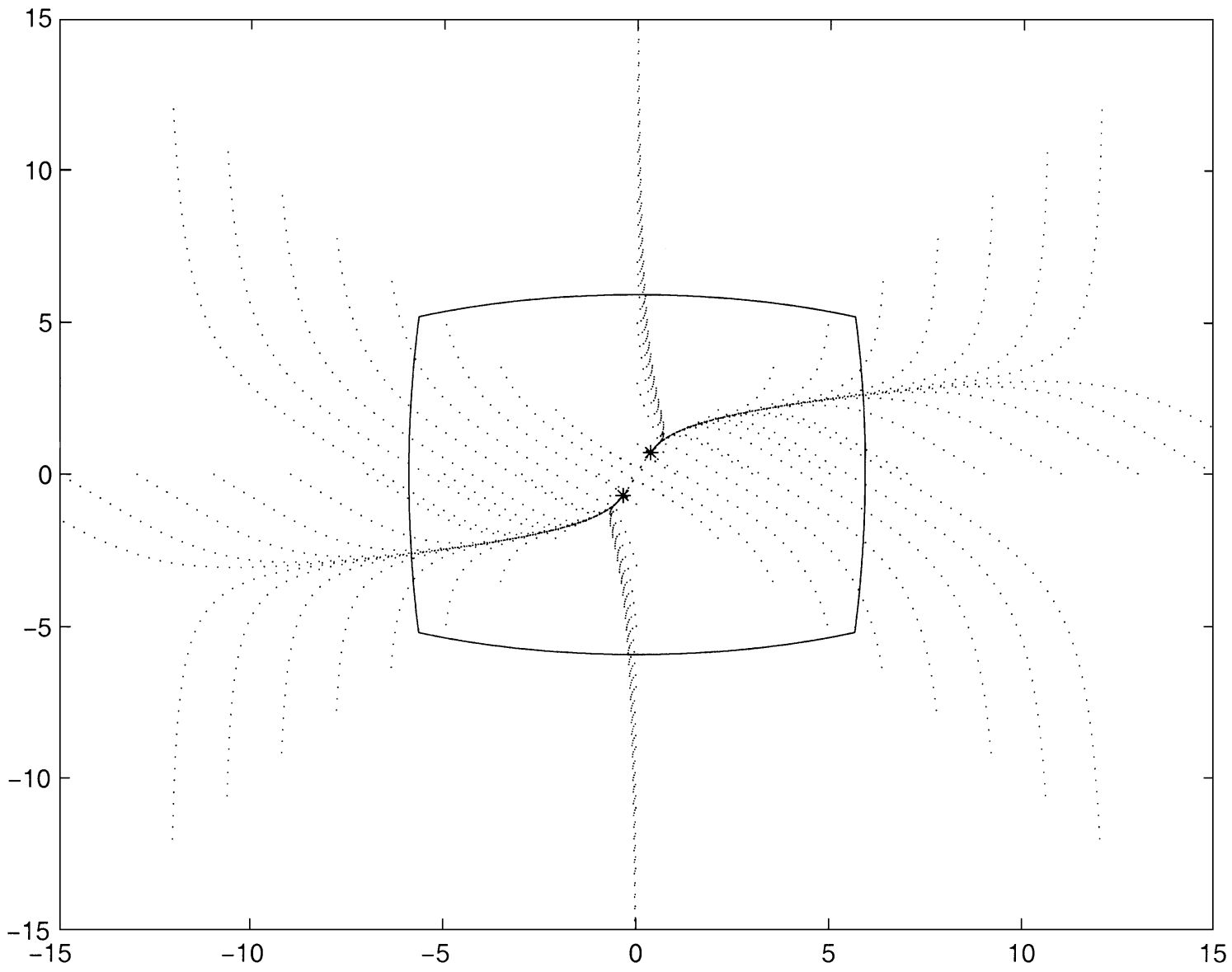

Fig. 2. Boundary of $S$ in plain line, $*$ the two equilibriums.

\section{CONCLUSION}

This note presents an extension of the small-gain theorem, using generalized $L_{2}$ gain instead of usual (constant) $L_{2}$ gain. It is shown that global asymptotic stability with no input can be guaranteed even when the product of the two $L_{2}$ gains (in the usual sense) is equal to one. Furthermore, convergence toward a bounded set can be guaranteed even when this product is larger than one.

Further results providing a characterization of the smallest generalized $L_{2}$ gains should lead to sharper bounds.

\section{REFERENCES}

[1] D. Angeli, E. Sontag, and Y. Wang, "Further equivalences and semiglobal versions of integral input to state stability," Dyna. Control, vol. 10 , no. 2, pp. 127-149, 2000

[2] A. Astolfi and A. Rapaport, "Robust stabilization of the angular velocity of a rigid body," Syst. Control Lett., vol. 34, pp. 257-264, 1998.

[3] C. A. Desoer and M. Vidyasagar, Feedback Systems: Input-Output Properties. New York: Academic, 1975.

[4] R. Freeman and P. Kokotovic, "Inverse optimality and robust stabilization," SIAM J. Control Optim., vol. 34, pp. 1365-1391, 1996.

[5] A. Isidori, Nonlinear Control Systems. London, U.K.: SpringerVerlag, 1995.

[6] A. Isidori and W. Lin, "Global $L_{2}$-gain design for a class of nonlinear systems," Syst. Control Lett., vol. 34, pp. 295-302, 1998.

[7] Z.-P. Jiang, I. Mareels, and Y. Wang, "A Lyapunov formulation of the nonlinear small-gain theorem for interconnected ISS systems," Automatica, vol. 32, no. 8, pp. 1211-1215, 1996.
[8] Y. Lin, E. Sontag, and Y. Wang, "Input to state stabilizability for parametrized families of systems," Int. J. Robust Nonlinear Control, vol. 5, pp. 187-205, 1995.

[9] M. R. James, "A partial differential inequality for dissipative nonlinear systems," Syst. Control Lett., vol. 21, pp. 315-320, 1993.

[10] A. Rapaport and A. Astolfi, "Practical $L_{2}$ disturbance attenuation for nonlinear systems," Automatica, vol. 38, no. 1, pp. 139-145, 2002.

[11] E. Sontag, "Comments on integral variants of ISS," Syst. Control Lett., vol. 34, pp. 93-100, 1998.

[12] E. Sontag and Y. Wang, "On characterizations of the input-to-state stability property. Output-to-state stability and detectability of nonlinear systems," Syst. Control Lett., vol. 24, pp. 351-359, 1995.

[13] _ - "On characterizations of input-to-state stability with respect to compact sets," in Proc. IFAC Non-Linear Control Systems Design Symp. (NOLCOS '95), Tahoe City, CA, 1995, pp. 226-231.

[14] _ - "Output-to-state stability and detectability of nonlinear systems," Syst. Control Lett., vol. 29, pp. 279-290, 1997.

[15] —-, "Lyapunov characterizations of input to output stability," SIAM J. Control Optim., vol. 39, pp. 226-249, 2000.

[16] A. Teel, "A nonlinear small gain theorem for the analysis of control systems with saturation," IEEE Trans. Automat. Control, vol. 41, pp. 1256-1270, July 1996.

[17] A. J. Van Der Schaft, $L_{2}$-Gain and Passivity Techniques in Nonlinear Control, ser. Lecture Notes in Control and Information Sciences. New York: Springer-Verlag, 1996, vol. 218, Lecture Notes in Control and Information Sciences.

[18] J. C. Willems, "Dissipative dynamical systems—Part I: General theory," Arch. Rational Mech. Anal., vol. 45, pp. 321-351, 1972. 